\title{
The distributed flight array
}

\section{Conference Paper}

\section{Author(s):}

Oung, Raymond; Bourgault, Frédéric; Donovan, Matthew; D'Andrea, Raffaello

Publication date:

2009

Permanent link:

https://doi.org/10.3929/ethz-a-005899689

Rights / license:

In Copyright - Non-Commercial Use Permitted 


\title{
The Distributed Flight Array
}

\author{
Raymond Oung, Frédéric Bourgault, Matthew Donovan, and Raffaello D’Andrea
}

\begin{abstract}
This paper introduces the Distributed Flight Array which is being developed at ETH Zurich. This multipropeller platform consists of autonomous single-propeller modules that are able to drive, dock with their peers, and fly in a coordinated fashion. These modules are organized as distributed computational units with minimal sensory input. This is a complex system that is rich in dynamics with much room to explore various strategies of distributed estimation and control. Several demonstrations in docking, driving, and flight have proven its feasibility.
\end{abstract}

\section{INTRODUCTION}

Vertical take-off and landing vehicles have gained popularity within the aerial vehicles community over the last few years. A particular advantage they have over most aerial vehicles is their unique ability for vertical stationary flight. Moreover, they provide an excellent platform for exploiting advanced sensor technology, pushing the limits of energy storage, and investigating techniques in automatic control.

Until now, researchers in this area have focussed primarily on quadrotor platforms [1], [2], [3], [4]. The Institute for Dynamic Systems and Control at ETH Zurich has extended this design to a multi-rotor platform with distributed control, which has been designated the Distributed Flight Array (DFA). The DFA consists of individual modules that are able to drive autonomously and assemble with their peers on the ground. Each module can generate enough thrust using a single fixed-pitch propeller to lift itself into the air, but is unstable in flight. Not until they are joined do these relatively simple modules evolve into a sophisticated multipropeller system capable of coordinated flight, see Fig. 1. The goal is to have many modules assemble at random, fly to a predetermined altitude, hover, break apart, fall back down, and repeat the cycle. This abstract testbed features rich dynamics and challenging design problems, and will undoubtedly be an eye-catching pedagogical showpiece for distributed estimation and control.

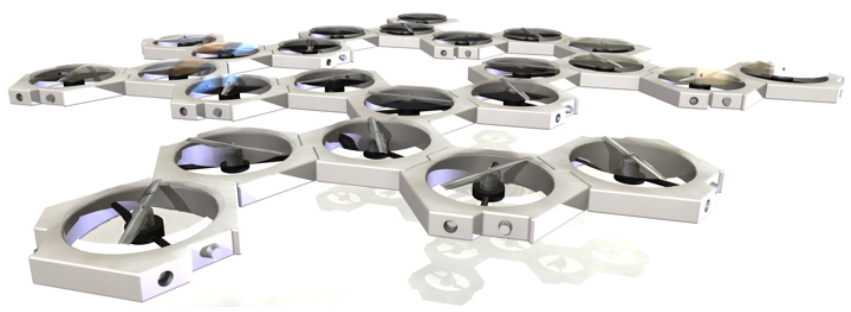

Fig. 1. This concept representation of the DFA is shown to be composed of a random arrangement of interconnected single-propeller modules that communicate with one another for coordinated flight.

The authors are with the Institute for Dynamic Systems and Control (IDSC), ETH Zurich, 8092 Zürich, Switzerland \{roung, fbourgault, mdonovan, rdandrea\} @ethz.ch.
This project comprises three key areas of interest: 1) Each DFA module must be optimized for weight, strength, and durability; 2) Modules must be able to drive and dock reliably with peers using a minimum number of sensors in order to reduce design complexity and energy usage; and 3) The DFA must be able to fly in a coordinated fashion regardless of the array's configuration.

This paper summarizes these three key aspects of the DFA and presents experimental results for docking, driving, and coordinated flight, the latter being the focus of recent publication [5]. This paper is organized as follows. Section II provides a detailed system description of a DFA module. Section III presents both the drive model and the flight model. Section IV briefly describes the docking strategy and Section V describes a simple distributed strategy for hover control. Experimental results are presented in Section VI. Concluding remarks are made in Section VII.

\section{DESIGN}

Experiments demonstrating feasibility of the DFA were recently completed using the first revision of the modules shown in Fig. 2(a), and it will be the focus of this paper. A second revision is currently under development, which will feature many improvements to the first revision, see Fig. 2(b).

The design challenges of the DFA mirror those of modular reconfigurable robots [6] and micro aerial vehicles [3], which include electromechanical interconnection, inter-module communication, and energy storage. The design of the system can be divided into four interconnected subsystems: (A) chassis \& docking mechanism; (B) drive unit; (C) flight unit; and (D) sensing, communication \& computation.

\section{A. Chassis \& Docking Mechanism}

Each DFA module resembles a hexagon with protruding features designed for passive alignment and docking, see Fig. 2(a). The mass of the chassis must be light enough to not impede flight, durable enough to withstand a drop from at least 2 meters, and robust enough to perform over a thousand cycles. To accomplish this, a low-density ethyl polypropylene (EPP) foam was chosen as the chassis material. Two-dimensional foam cut-outs were layered on top of one another to generate the assembly shown in Fig. 2(a). This simple manufacturing process saves time and cost. However, it does not allow the possibility of generating a smooth leading edge for the duct which could improve thrust efficiency.

The protruding features assist with alignment and eliminate the unnecessary complexity of an active docking mech- 


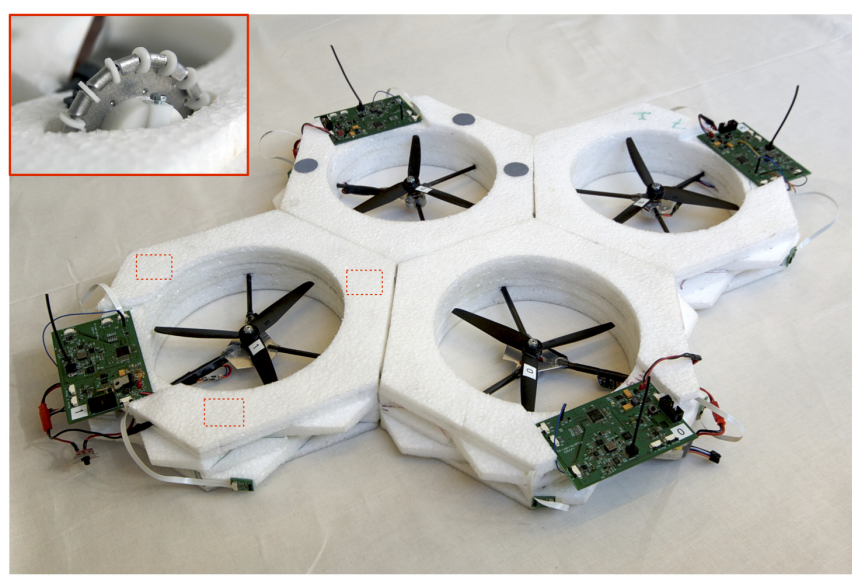

(a) Shown here are four prototype DFA modules (Rev. 1) in a docked configuration. The module's chassis resembles a hexagon with protruding features designed for passive alignment and docking; it is assembled from 2D low-density ethyl polypropylene (EPP) foam cut-outs. The dotted boxes indicate the location of the custom-made omni-wheels which are embedded in the foam and are used to drive the module.

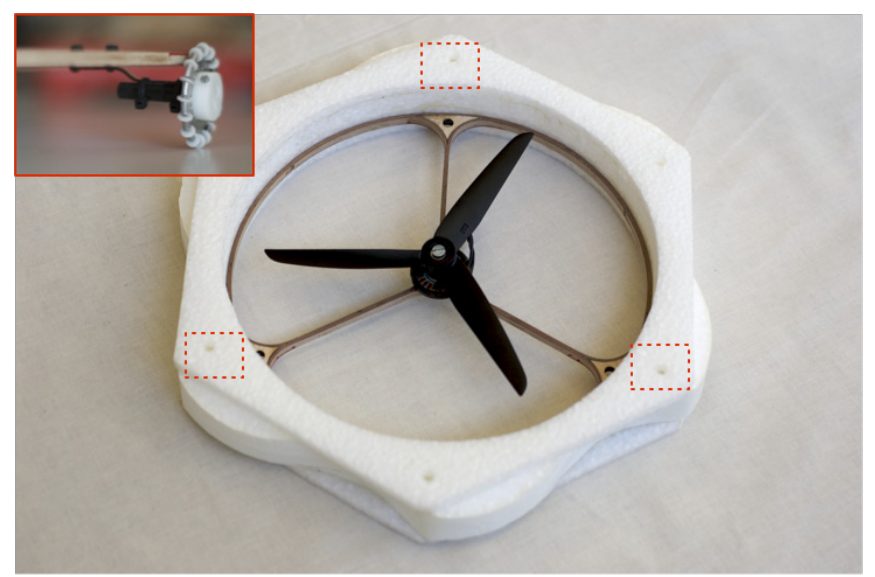

(b) Currently in development is the second revision of the DFA module. Seen in this photo is the 3-blade fixed-pitch propeller mounted to a brushless DC motor, a plywood molded frame, and the foam chassis. The on-board electronics will be embedded in the foam chassis for protection against impact; also embedded are the custom-made omni-wheels indicated by the dotted boxes. Each drive motor is mounted to a cantilever spring suspension in order to absorb shock upon impact and prevent damage to the drive motors.

Fig. 2. Development revisions of the DFA: (a) revision 1 is presented in this paper, and (b) revision 2 is currently being developed.

anism. Keeping the modules attached to one another is a symmetric arrangement of four permanent magnets on each side of the module. The magnets have been chosen to be strong enough to keep the modules together and to withstand the stresses of flight, but weak enough to break apart when sufficient force and/or torque is applied by a module.

Table I lists some of the important physical attributes which characterize a DFA module.

\section{B. Drive Unit}

Mounted to three sides of the chassis are custom-made omni-wheels with rollers orthogonal to the axis of the wheel, see insets of Fig. 2. A 0.5 Watt brushed DC motor with integrated encoder for velocity feedback drives each wheel. Omni-wheels were chosen because they offer a high degree of maneuverability in plane, and they eliminate any steering
TABLE I

Physical Attributes of a DFA Module (ReV. 1)

\begin{tabular}{l|c|c|}
\hline Symbol & Description & Value \\
\hline \hline$\ell$ & Characteristic length $\dagger$ & $0.250 \mathrm{~m}$ \\
\hline$r_{w}$ & Wheel distance $\dagger \dagger$ & $0.100 \mathrm{~m}$ \\
\hline- & Propeller duct diameter & $0.180 \mathrm{~m}$ \\
\hline$m$ & Mass per module & $0.180 \mathrm{~kg}$ \\
\hline$\dagger$ Defined as the distance between opposite sides of a module \\
$\dagger \dagger$ Defined as the distance from the module's center to the wheel
\end{tabular}

linkage(s) that would otherwise be necessary for coordinated driving, as will be demonstrated later.

\section{Flight Unit}

Mounted to the center of the chassis is a 50 Watt brushless DC motor with an off-the-shelf electronic speed controller and a 3-blade fixed-pitch propeller capable of producing more than 3 Newtons of thrust. Embedded in the chassis is a Lithium-Ion Polymer battery that is capable of powering both the motors and the electronics for up to 5 minutes of flight. All modules are identical except for the propeller orientation, where there are two possible orientations: clockwise (CW) and counterclockwise (CCW). This is necessary to cancel the aerodynamic torques in trimmed flight.

\section{Sensing, Communication \& Computation}

Custom-designed electronics were made to meet all the on-board sensing, communication, and computation requirements. Each module comes equipped with a 3-axis rate gyro for measuring angular rates and a pressure sensor for measuring altitude. Bi-directional inter-module communication is accomplished with IrDA via a UART peripheral; there is an IrDA transceiver mounted to each side of the module. An ARM7 core microcontroller handles all the required computation needed for estimation and control.

\section{MODELING}

\section{A. Kinematic Drive Model}

Since the modules drive on the ground with relatively low velocities, dynamic effects that act on the system may be neglected. For this reason, the drive model considers only the kinematics of a rigid body with three independently-driven omni-wheels.

The module's body coordinate frame $M$ coincides with the center of the module, see Fig. 3. It is oriented such that the $x$-axis is pointed perpendicular to a side of the module, and the $y$-axis is $90^{\circ} \mathrm{CCW}$ from the $x$-axis in the plane of the module. The transformation matrix $\mathbf{J}$ which maps the module's velocity $\dot{\zeta}=\left(V_{x}, V_{y}, \dot{\theta}\right)$ in the module's body coordinate frame to wheel velocities $\mathbf{v}=\left(v_{1}, v_{2}, v_{3}\right)$ for an omni-directional vehicle has been developed in previous work [7] and will only be summarized here:

$$
\mathbf{v}=\mathbf{J} \dot{\zeta}
$$

where

$$
\mathbf{J}=\left[\begin{array}{ccc}
-\frac{\sqrt{3}}{2} & \frac{1}{2} & r_{w} \\
0 & -1 & r_{w} \\
\frac{\sqrt{3}}{2} & \frac{1}{2} & r_{w}
\end{array}\right]
$$



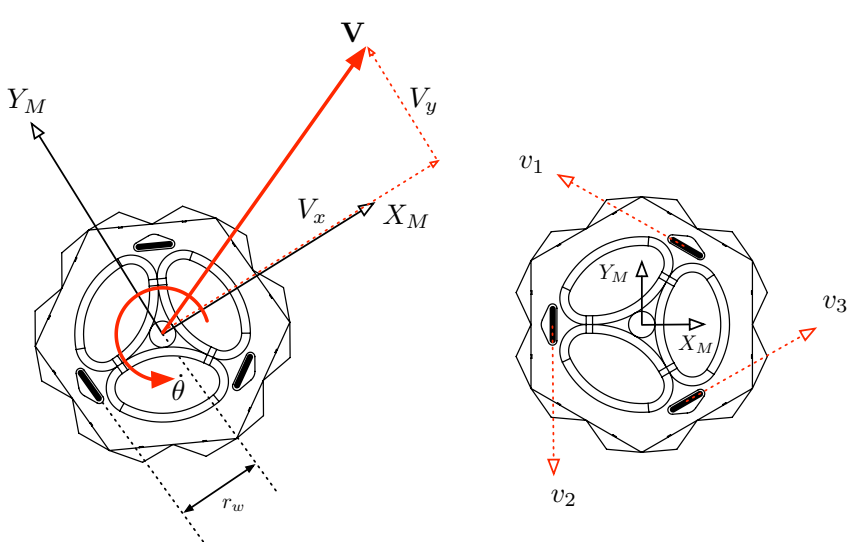

Fig. 3. The DFA module's drive model consists of a rigid body with three independently driven omni-wheels. Shown in this diagram is the body coordinate frame of the module and the velocity vectors.

and $r_{w}$ is the distance of the wheel from the center of the module, see Fig. 3.

\section{B. Dynamic Flight Model}

The full flight dynamics of the DFA can be quite complex if effects like the flexibility of the propellers [8], aerodynamic effects of the propeller duct, and the forces that keep the modules together are considered. As a first step, the system is simply modeled as a rigid body without any compliant inter-module connections, incorporating a force and torque generation process at each module around the hovering equilibrium. This will be shown to be adequate for the purpose of hover control.

The DFA's body coordinate frame $B$ coincides with the array's center of mass and is aligned with its principal axes of rotation, see Fig. 4. A sequence of three rotations described by the Euler angles $\alpha, \beta, \gamma$ acting along the $z-, y-, x-$ axis in this order, describes the orientation of the DFA's body coordinate frame with respect to the inertial coordinate frame.

Let $\left(\mathrm{x}_{i}, \mathrm{y}_{i}\right)$ be the coordinate location of module $i$ with respect to the DFA's body coordinate frame. The altitude and attitude of the DFA can be controlled by varying the force (or thrust) $f_{i}$ and torque $\tau_{i}$ produced by each module, see Fig. 4. How these control forces are generated will be described later. The total thrust generated by $N$ modules is the sum of all thrusts produced by each module, $F=\sum_{i=1}^{N} f_{i}$. The rolling torque is the sum of all thrusts acting along the moment arm $\mathrm{y}_{i}, T_{\gamma}=\sum_{i=1}^{N} \mathrm{y}_{i} f_{i}$. Similarly, the pitching torque is the sum of all thrusts acting along the moment arm $\mathrm{x}_{i}, T_{\beta}=-\sum_{i=1}^{N} \mathrm{x}_{i} f_{i}$. The yawing torque is the sum of all reaction torques produced by each module; in this case, torque can be accurately modeled as a linear function of thrust [5]. Hence, the yawing torque can be expressed as $T_{\alpha}=\sum_{i=1}^{N} c_{i} f_{i}$, where the sign of $c$ depends on the rotation of the propeller: the sign is positive when the propeller rotates $\mathrm{CCW}$ and negative when the propeller rotates $\mathrm{CW}$.

The following summarizes the equations of motion which are linearized about hover and are normalized in order to gain some intuition about how the size of the array $N$ affects flight dynamics [5]:

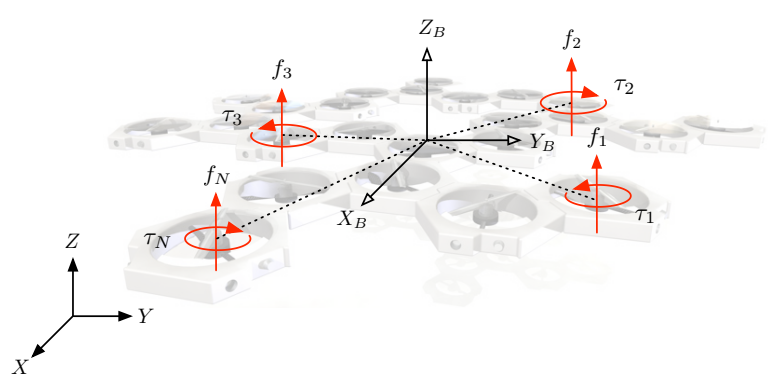

Fig. 4. Shown in this diagram is the inertial coordinate frame and the DFA's body coordinate frame $B$ with force/torque vectors produced by various modules. The body coordinate frame is chosen to coincide with the array's center of mass and is aligned with the principal axes of rotation.

$$
\begin{aligned}
\ddot{z} & =\frac{1}{N} \sum_{i=1}^{N} a_{i} \\
\hat{I}_{x} \ddot{\gamma} & =\frac{1}{N} \sum_{i=1}^{N} \hat{\mathrm{y}}_{i} a_{i} \\
\hat{I}_{y} \ddot{\beta} & =-\frac{1}{N} \sum_{i=1}^{N} \hat{\mathrm{x}}_{i} a_{i} \\
\hat{I}_{z} \ddot{\alpha} & =\frac{1}{N} \sum_{i=1}^{N} \hat{c}_{i} a_{i}
\end{aligned}
$$

where

$$
\hat{\mathrm{x}}_{i}=\frac{\mathrm{x}_{i}}{\frac{\ell \sqrt{N}}{2}}, \quad \hat{\mathrm{y}}_{i}=\frac{\mathrm{y}_{i}}{\frac{\ell \sqrt{N}}{2}}, \quad \hat{c}_{i}=\frac{c_{i}}{\ell}
$$

Note that $\hat{\mathrm{x}}_{i}$ and $\hat{\mathrm{y}}_{i}$ are normalized position coordinates and are at most on the order of 1 for a disc-like array, $\hat{c}_{i}$ is the normalized force to torque conversion constant and is expected to be much less than 1 , and $a_{i}$ is the normalized control input in units of acceleration. The normalized principal mass moments of inertia $\left(\hat{I}_{x}, \hat{I}_{y}, \hat{I}_{z}\right)$ can be written as the following:

$$
\hat{I}_{x}=\frac{\epsilon_{x} \ell \sqrt{N}}{8}, \quad \hat{I}_{y}=\frac{\epsilon_{y} \ell \sqrt{N}}{8}, \quad \hat{I}_{z}=\frac{\epsilon_{z} \ell N}{8}
$$

where $\left(\epsilon_{x}, \epsilon_{y}, \epsilon_{z}\right)$ captures the mass distribution of the array.

It can be seen from the equations above that the maximum vertical acceleration $\ddot{z}$ is independent of $N$. For disc-like arrays, the maximum accelerations in roll $\ddot{\gamma}$ and in pitch $\ddot{\beta}$, however, decrease by a factor of $\sqrt{N}$, while the maximum acceleration in yaw $\ddot{\alpha}$ decreases by a factor of $N$.

Note that the normalized control inputs $a_{i}$ are not directly generated. The input to the module's flight system is a pulse width modulated (PWM) duty cycle $D$, which effectively controls the angular velocity of the rotor. The output from this system is both the generated force and torque of the module. Experimental results show that force and duty cycle can be approximated by an affine relationship around the hovering thrust, while the torque resulting from the propeller's drag can be approximated as a linear function of thrust [5].

Motivated by these results, the transfer function $G_{f}$ which relates the input desired thrust $f(D)$ to the output thrust 
is modeled as a linear time-invariant system. The transfer function that was obtained from the Bode plot of the thrust response, shown in Fig. 5, was found to approximate a firstorder system:

$$
G_{f}(s)=\frac{\omega}{s+\omega}
$$

where $\omega=14.3 \mathrm{rad} / \mathrm{s}$.
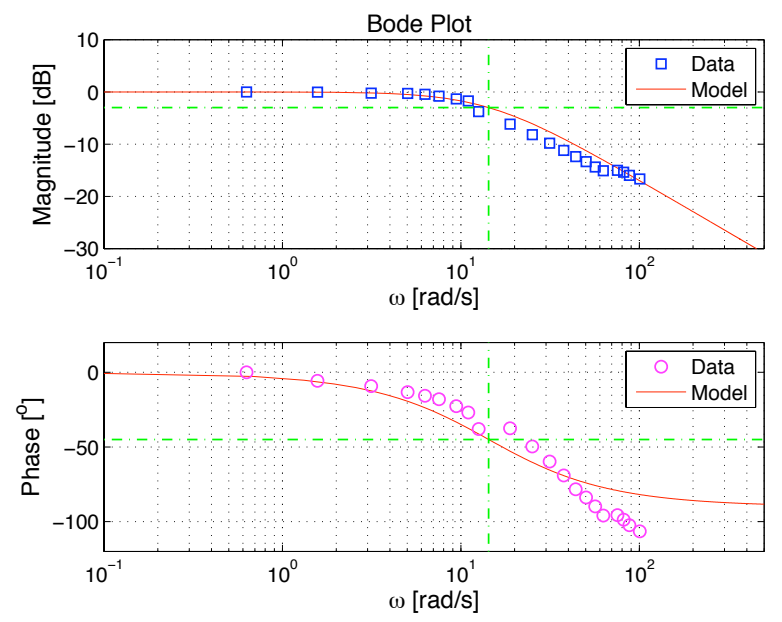

Fig. 5. The Bode plot of the thrust response is shown here to approximate a first-order system. Sinusoidal varying duty cycle with a bias equal to the equilibrium thrust and an amplitude equivalent to $0.5 \mathrm{~N}$ was sent to the system. Force and torque measurements were made using a 6-axis forcetorque strain gauge load cell. The dash-dotted lines in the plots represent the $-3 \mathrm{~dB}$ frequency, which is $14.3 \mathrm{rad} / \mathrm{s}$, and the corresponding magnitude and phase at this frequency. Note that at high frequencies the measured phase diverges from the model; this is due to unmodeled dynamics, such as delays.

\section{DOCKING STRATEGY}

At the start of each cycle, a number of DFA modules will be scattered randomly across the floor. The objective for each module will be to dock with one another in preparation for coordinated flight. Although there are many ways in which this can be accomplished, one particular strategy described in [9] is summarized here.

Consider an overhead light source as the only environmental feature detectable by each module via a single on-board photodiode. This photodiode along with encoder odometry will be the only source of information used for pose estimation. Modules will be able to move around a prescribed circular region as defined by the cone of illumination from the overhead light source, see Fig. 6. A user-defined light intensity threshold will force a module to be in one of two states: (1) within the circular region and (2) outside the circular region.

Without any prior knowledge about the environment, the objective of each module is to drive to the center of the circular region and to dock with their peers. A simple strategy could be to have each module drive in a random direction until it crosses the boundary of the circular region at three different locations. These three data points could then be used to estimate the center of the circle. As modules randomly interconnect during the data point collection process, they can share information to improve their common estimate of the circular region. Depending on their estimate, they will coordinate with each other to continue exploration or to move towards the center.

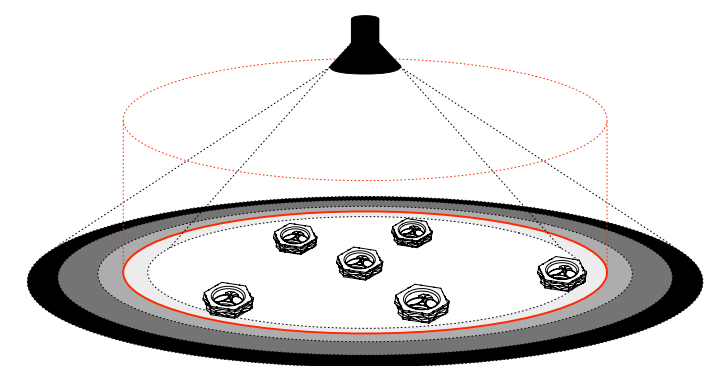

Fig. 6. A set of six DFA modules randomly scattered across a circular arena defined by the cone of illumination from an overhead light source. The perimeter of this circular region can be adjusted by varying the light intensity, the intensity threshold of the photodiode, the height of the lamp, and/or the aperture of the light source.

A 2D simulator using the Box2D physics engine [10] is currently being developed to experiment with this docking strategy, see Fig. 7. It will be used to obtain array configuration statistics, which will be needed to determine the typical values of the mass distribution parameters $\left(\epsilon_{x}, \epsilon_{y}, \epsilon_{z}\right)$ required for testing various control strategies and analyzing their performance.

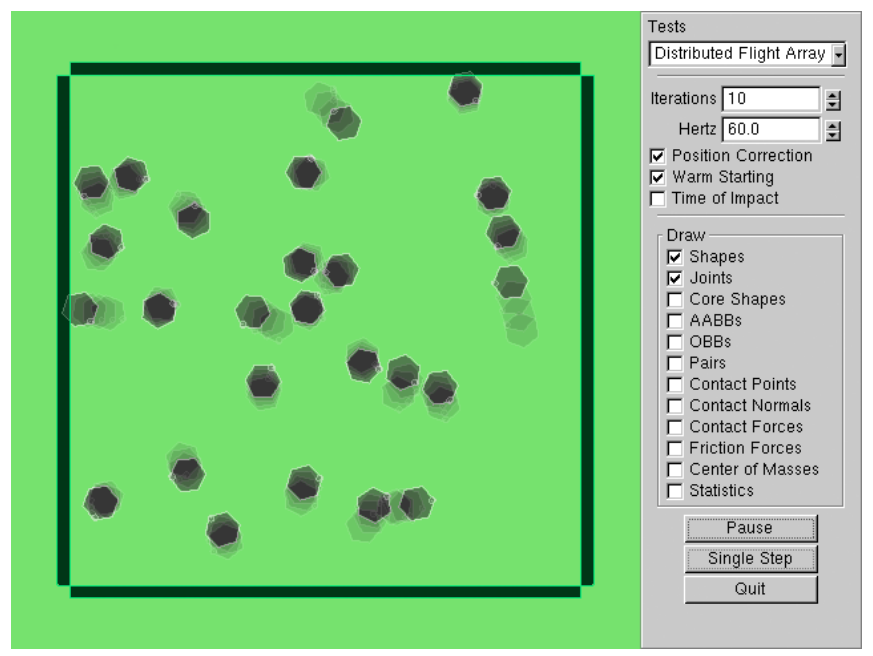

Fig. 7. A docking/driving simulator is being developed for the DFA using the Box2D physics engine. The simulator will be used to obtain array configuration statistics, which will be needed in testing various control strategies and analyzing their performance.

\section{Flight CONTROL}

This section presents a simple distributed strategy for hover control based on physical parameters of the DFA. A reason for using a distributed strategy is that it can be made scalable and robust to module failure [11]. The control strategy presented here is generalized and assumes full state feedback of the system, see Eq. 6. It is assumed that an estimator is used to obtain the state of the system. 
Starting with the dynamic model of the DFA presented in the previous section, the normalized and linearized equations of motion about the equilibrium, Eq. 1-4, can be written as

$$
\mathbf{M} \ddot{\mathbf{s}}=\mathbf{P}^{T} \mathbf{a}
$$

where

$$
\begin{aligned}
\mathbf{M} & =\operatorname{diag}\left(1, \hat{I}_{x}, \hat{I}_{y}, \hat{I}_{z}\right) \\
\mathbf{s} & =[z, \gamma, \beta, \alpha]^{T} \\
\mathbf{a} & =\left[a_{1}, \ldots, a_{N}\right]^{T}
\end{aligned}
$$

The matrix $\mathbf{P}$ contains information pertaining to the configuration of the array and can be written as

$$
\mathbf{P}=\left[\mathbf{p}_{z}, \mathbf{p}_{\gamma}, \mathbf{p}_{\beta}, \mathbf{p}_{\alpha}\right]
$$

where

$$
\begin{aligned}
\mathbf{p}_{z} & =\frac{1}{N}[1, \ldots, 1]^{T} & \mathbf{p}_{\gamma} & =\frac{1}{N}\left[\hat{\mathrm{y}}_{1}, \ldots, \hat{\mathrm{y}}_{N}\right]^{T} \\
\mathbf{p}_{\beta} & =-\frac{1}{N}\left[\hat{\mathrm{x}}_{1}, \ldots, \hat{\mathrm{x}}_{N}\right]^{T} & \mathbf{p}_{\alpha} & =\frac{1}{N}\left[\hat{c}_{1}, \ldots, \hat{c}_{N}\right]^{T}
\end{aligned}
$$

Consider the control strategy of the following form:

$$
\mathbf{a}=\mathbf{Q} f(z, \dot{z}, \gamma, \dot{\gamma}, \beta, \dot{\beta}, \alpha, \dot{\alpha})
$$

where

$$
\begin{aligned}
\mathbf{Q} & =\left[\mathbf{q}_{z}, \mathbf{q}_{\gamma}, \mathbf{q}_{\beta}, \mathbf{q}_{\alpha}\right] \\
f & =\left[f_{z}(z, \dot{z}), f_{\gamma}(\gamma, \dot{\gamma}), f_{\beta}(\beta, \dot{\beta}), f_{\alpha}(\alpha, \dot{\alpha})\right]^{T}
\end{aligned}
$$

where the $f .(\cdot)$ are arbitrary functions, to be determined.

It can be shown that for a large and equal number of $\mathrm{CW}$ and CCW modules, one could use the following elements of $\mathbf{Q}$ to decouple the degrees of freedom [5]:

$$
\begin{aligned}
\mathbf{q}_{z} & =\frac{\mathbf{p}_{z}}{\left\|\mathbf{p}_{z}\right\|^{2}} & \mathbf{q}_{\gamma} & =\frac{\mathbf{p}_{\gamma}}{\left\|\mathbf{p}_{\gamma}\right\|^{2}} \\
\mathbf{q}_{\beta} & =\frac{\mathbf{p}_{\beta}}{\left\|\mathbf{p}_{\beta}\right\|^{2}} & \mathbf{q}_{\alpha} & =\frac{\mathbf{p}_{\alpha}}{\left\|\mathbf{p}_{\alpha}\right\|^{2}}
\end{aligned}
$$

Note that the elements of $\mathbf{Q}$ above are not a function of $N$, making the decoupling strategy independent of $N$.

This control strategy only works if both $\mathbf{P}$ and $\left(\hat{I}_{x}, \hat{I}_{y}, \hat{I}_{z}\right)$ are known. Both of these can be computed if the position and the direction of propeller rotation for each module is known; this assumes that all modules are identically the same, and that the mass and mass moments of inertia are given. It follows that position and the direction of propeller rotation is the only information that needs to be communicated across the array before taking flight.

With a decoupled control strategy, one can consider each degree of freedom separately. For example, the following functions can be chosen:

$$
\begin{aligned}
f_{z}(z, \dot{z}) & =-2 \omega_{z} \zeta_{z} \dot{z}-\omega_{z}^{2}\left(z-z_{d}\right) \\
f_{\gamma}(\gamma, \dot{\gamma}) & =-\hat{I}_{x}\left(2 \omega_{\gamma} \zeta_{\gamma} \dot{\gamma}+\omega_{\gamma}^{2} \gamma\right) \\
f_{\beta}(\beta, \dot{\beta}) & =-\hat{I}_{y}\left(2 \omega_{\beta} \zeta_{\beta} \dot{\beta}+\omega_{\beta}^{2} \beta\right) \\
f_{\alpha}(\alpha, \dot{\alpha}) & =-\hat{I}_{z}\left(2 \omega_{\alpha} \zeta_{\alpha} \dot{\alpha}+\omega_{\alpha}^{2} \alpha\right)
\end{aligned}
$$

where each degree of freedom is a second-order system with two tuning parameters: $\left(\omega_{z}, \omega_{\gamma}, \omega_{\beta}, \omega_{\alpha}\right)$ representing the natural frequencies of the system and $\left(\zeta_{z}, \zeta_{\gamma}, \zeta_{\beta}, \zeta_{\alpha}\right)$ representing the damping ratios. The variable $z_{d}$ represents the desired hovering altitude.

This control strategy uses normalized thrust as the control input, which is not the case as described in Section III-B. Thrust dynamics and saturation of the control inputs should be considered. Time-scale separation is needed between the desired dynamics of the system and the rotor dynamics. A way to achieve this is to invert the transfer function $G_{f}$ over a desired frequency range, enough to achieve time-scale separation.

This control strategy has been simulated in MATLAB for random array configurations consisting of up to 20 modules, see Fig. 8. Simulation experiments take into account sensor noise derived from physical experiments and the motor model described in Section III-B.

\section{EXPERIMENTS}

Three important aspects of the DFA were demonstrated in order to verify its feasibility ${ }^{1}:$ 1) Docking, 2) Driving, and 3) Flying. Each one in sequence is a prerequisite to the next, and therefore each is needed to achieve the end goal of coordinated flight.

\section{A. Docking}

Experiments were performed to test the module's ability to interconnect. Statistics were gathered from experiments where two modules drove into each other at various angles and from experiments where one module rotated in place while another drove into it. Based on these experimental results, two modules have the highest probability of successfully docking when one is rotating and another is translating along converging trajectories, see Fig. 9. This is an important observation to consider when developing the docking strategy.

The driving surface also plays a critical role in enabling the modules to dock. The surface must be flat and be sufficiently tacky to prevent the wheels from slipping while driving, but allow for slippage when docking. A non-stick Polyvinyl Chloride (PVC) closed-cell foam material, similar to that used in Yoga mats, was found to work effectively.

\section{B. Driving}

A pair of modules was demonstrated to drive together and perform maneuvers which would otherwise be impossible without the use of omnidirectional wheels or a steering mechanism. Once docked together, the modules synchronized themselves via IrDA then performed pirouettes along a circular trajectory, i.e. the pair of modules rotated about their combined center while both modules followed a 2 meter diameter circular trajectory in the direction opposite to their rotation.

\footnotetext{
${ }^{1}$ A video summary of the DFA which demonstrates docking, driving, and coordinated flight:

http://www.tinyurl.com/distributed-flight-array
} 
CONFIDENTIAL. Limited circulation. For review only.

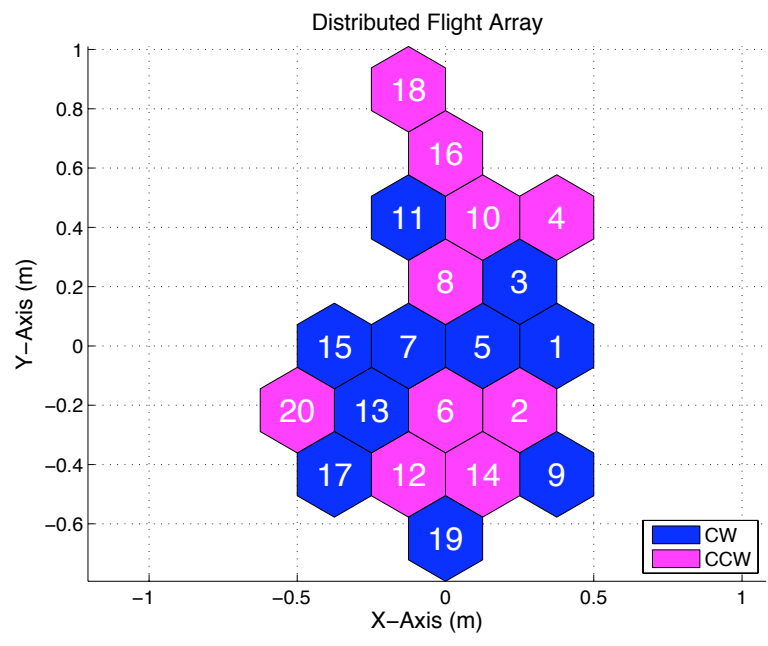

(a)
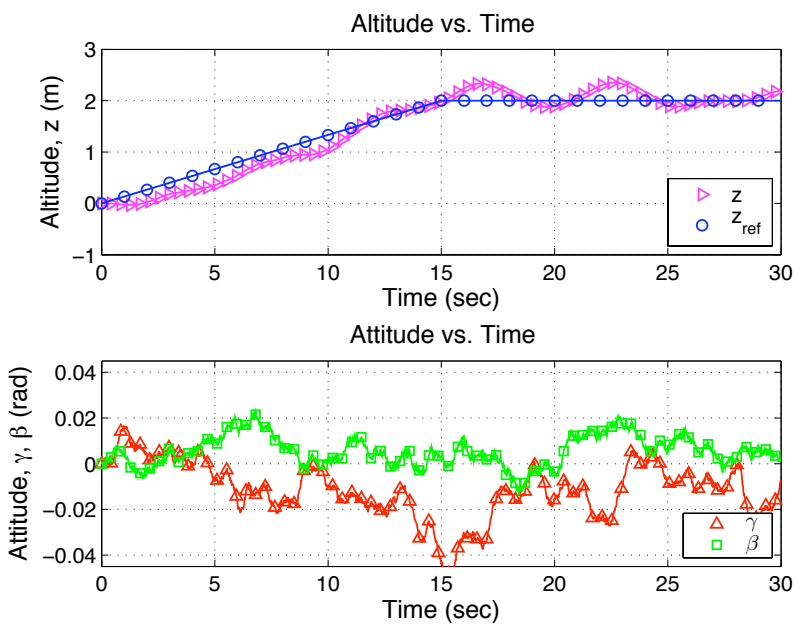

(b)

Fig. 8. Simulation results for a 20 -module configuration, where the natural frequencies were set to $\left(\omega_{z}, \omega_{\gamma}, \omega_{\beta}, \omega_{\alpha}\right)=(0.67,5.81,5.81,0.45)$ and the damping ratios were set to $\left(\zeta_{z}, \zeta_{\gamma}, \zeta_{\beta}, \zeta_{\alpha}\right)=(1,1,1,1)$.
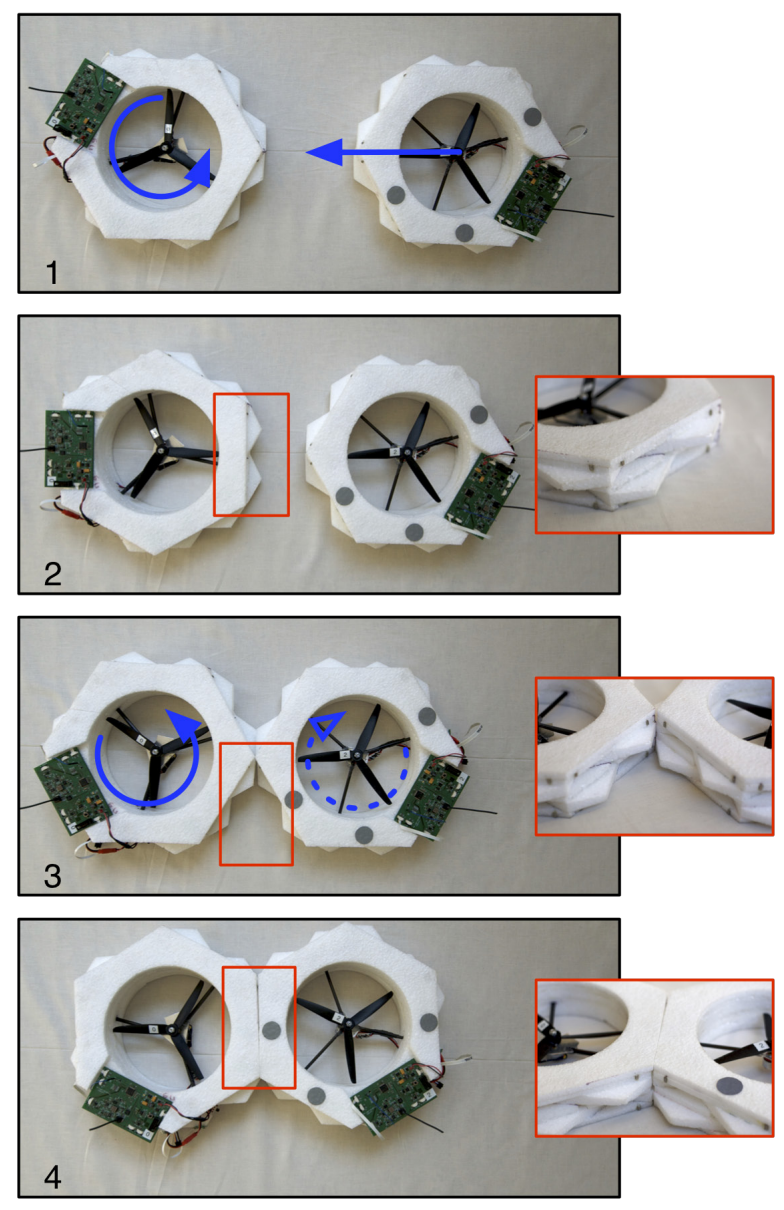

Fig. 9. Shown here is an image sequence of two DFA modules docking. In frame 1 , the left module rotates $\mathrm{CCW}$ while the right module drives towards the rotating module. In frame 3, the modules collide. The left module continues to rotate $\mathrm{CCW}$ and due to its protrusions it induces a $\mathrm{CW}$ rotation in the right module. The modules finally dock together, making a solid interface in frame 4.

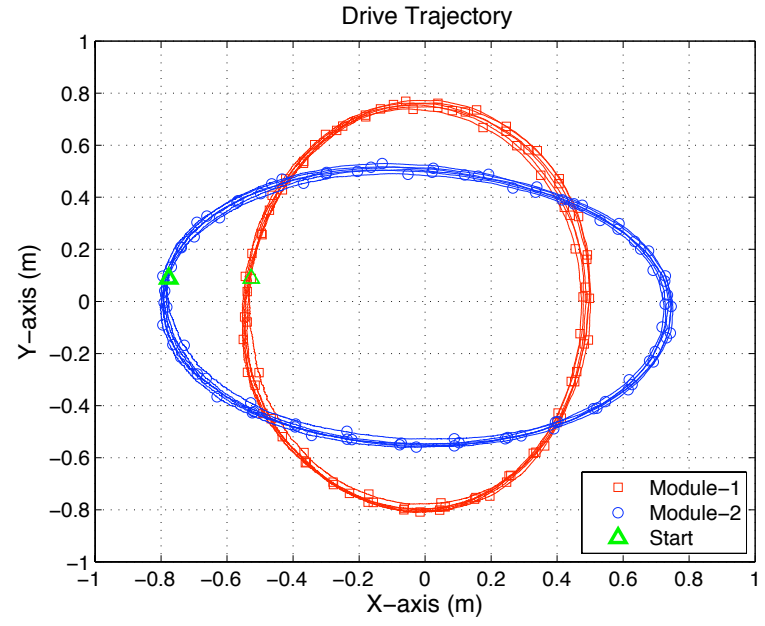

Fig. 10. Shown here is a plot of the trajectory made by a pair of modules during a pirouette maneuver, measured using a Vicon MX system. Note that the maneuver is very repeatable for over six orbits considering that there is no feedback on the pose of either modules.

A Vicon MX system [12] was used to measure the performance of this pirouette maneuver, which performed very well considering that there was no feedback on the pose of the two modules, see Fig. 10. The pair was able to meet the desired trajectory with relatively good accuracy and precision while performing a 1.3 meter diameter orbit over 6 times.

\section{Flying}

The linear model presented in Section III-B and the results gathered from simulating the controller described in Section V were verified by testing the control strategy on the DFA in the array configuration shown in Fig. 2(a). Initial experiments established that the pressure sensor performed poorly and provided imprecise altitude measurements. Moreover, yaw control was intentionally left out to simplify the 
experiments. Consequently, experiments were made using only roll and pitch as feedback to the controller, see Eq. 8-9.

Before taking flight, the modules synchronized themselves via IrDA and calibrated their sensors by removing sensor offsets over a 5 second initialization sequence. Rate-gyro measurements were made at $200 \mathrm{~Hz}$ and the controller was operated at $60 \mathrm{~Hz}$. The control input $f_{z}$ was set to a very small value. A Vicon MX system was used to measure both altitude and attitude of the DFA, see Fig. 11(b)-11(c).

The DFA was shown to fly successfully with roll and pitch control. The experimental results shown in Fig. 11(a)11(b) look promising as they are comparable to the simulated system, thus verifying the utility of the linear model and simulator.

\section{CONClusions AND Future Work}

This paper presented the Distributed Flight Array (DFA), a unique modular multi-rotor vehicle capable of autonomous self-assembly and coordinated flight. The initial prototype of the DFA has demonstrated various important aspects of the system, including: docking, driving, and flying. The DFA is currently undergoing its second revision which will enable coordinated driving and flying experiments on a larger scale. These results will be presented in future work.

\section{REFERENCES}

[1] J. How, B. Bethke, A. Frank, D. Dale, and J. Vian, "Real-time indoor autonomous vehicle test environment," Control Systems Magazine, IEEE, vol. 28, no. 2, pp. 51-64, April 2008.

[2] G. Hoffmann, H. Huang, S. Waslander, and C. Tomlin, "Quadrotor helicopter flight dynamics and control: Theory and experiment," in Proceedings of the AIAA Guidance, Navigation, and Control Conference, Aug. 2007.

[3] S. Bouabdallah, M. Becker, and R. Siegwart, "Autonomous miniature flying robots: coming soon! - research, development, and results," Robotics \& Automation Magazine, IEEE, vol. 14, no. 3, pp. 88-98, Sept. 2007.

[4] N. Guenard, T. Hamel, and V. Moreau, "Dynamic modeling and intuitive control strategy for an "x4-flyer"," in Proceedings of the IEEE International Conference on Control and Automation, vol. 1, June 2005, pp. 141-146.

[5] R. Oung, A. Ramezani, and R. D'Andrea, "Feasibility of a distributed flight array," in IEEE Conference on Decision and Control (to appear), 2009.

[6] M. Yim, W.-M. Shen, B. Salemi, D. Rus, M. Moll, H. Lipson, E. Klavins, and G. Chirikjian, "Modular self-reconfigurable robot systems [grand challenges of robotics]," Robotics \& Automation Magazine, IEEE, vol. 14, no. 1, pp. 43-52, March 2007.

[7] G. Campion, G. Bastin, and B. Dandrea-Novel, "Structural properties and classification of kinematic and dynamic models of wheeled mobile robots," Robotics and Automation, IEEE Transactions on, vol. 12, no. 1, pp. 47-62, Feb 1996.

[8] P.-J. Bristeau, P. Martin, E. Salaun, and N. Petit, "The role of propeller aerodynamics in the model of a quadrotor uav," in European Control Conference, 2009. ECC., 2009.

[9] F. Bourgault and R. D'Andrea, "Distributed localization and navigation in a circular arena for assembly of a distributed array," in IEEE International Conference on Robotics and Automation (submitted), 2010.

[10] "Box2d physics engine homepage," Sept. 2009, [Accessed: Sept. 7, 2009]. [Online]. Available: http://www.box2d.org/

[11] E. Klavins, "Programmable self-assembly," Control Systems Magazine, IEEE, vol. 27, no. 4, pp. 43-56, Aug. 2007.

[12] "Vicon mx homepage," Aug. 2009, [Accessed: Aug. 31, 2009]. [Online]. Available: http://www.vicon.com/products/viconmx.html
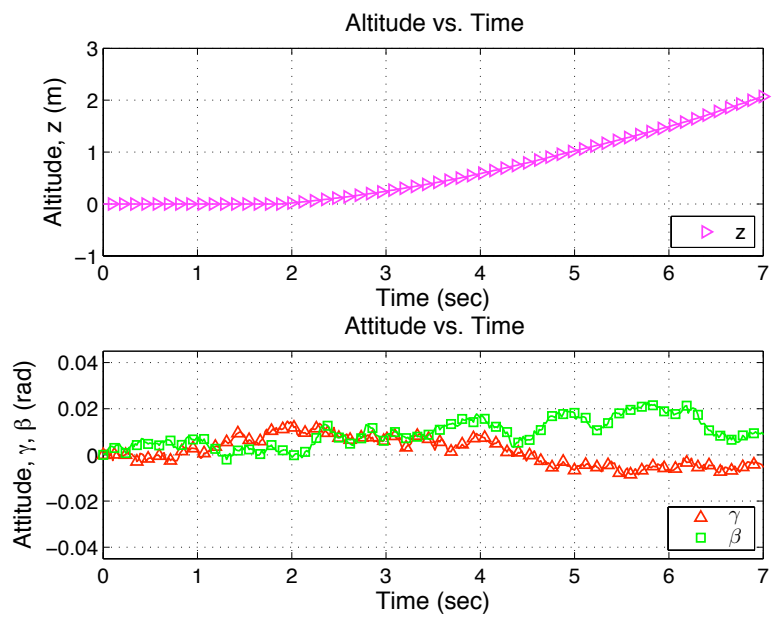

(a) Simulated results for a 4-module configuration without any feedback on altitude, where the natural frequencies were set to $\left(\omega_{\gamma}, \omega_{\beta}\right)=(13,13)$ and the damping ratios were set to $\left(\zeta_{\gamma}, \zeta_{\beta}\right)=(1,1)$.
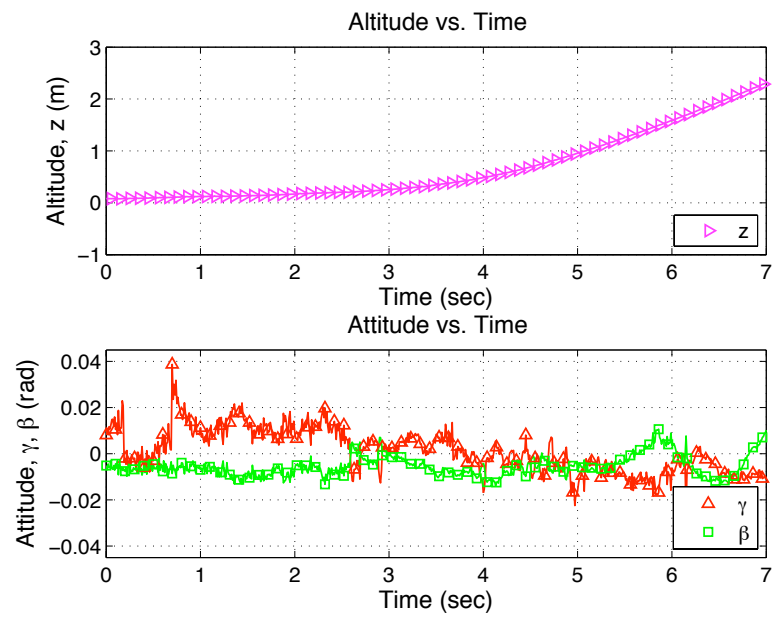

(b) The controller that was simulated with results shown in (a), was also used in an experimental flight test; the measurements obtained from this test are shown here.

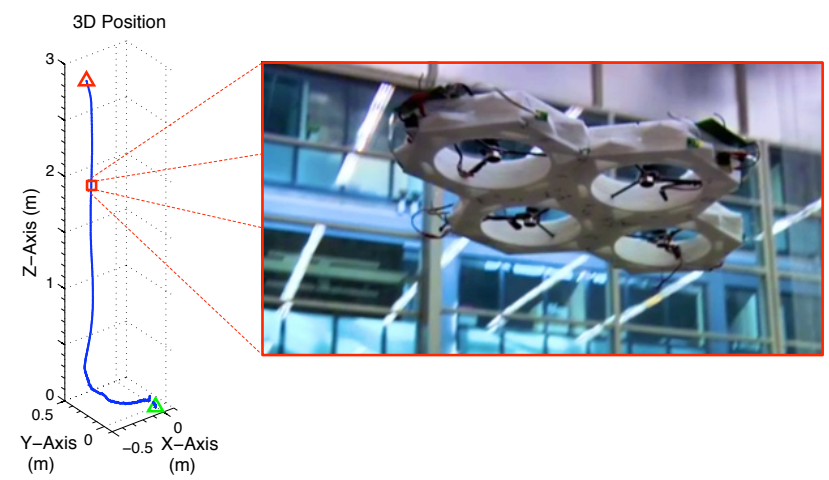

(c) A plot of the DFA's 3D position relative to its take-off origin, obtained from experiments.

Fig. 11. Shown here is a comparison of the results gathered from a simulation and an experimental flight test without any feedback on altitude. Experimental measurements were made using a Vicon MX system. 